\title{
Nanotechnology: Pediatric Applications
}

\author{
MARY C. MACHADO, DANIEL CHENG, KEIKO M. TARQUINIO, AND THOMAS J. WEBSTER
}

\author{
Division of Engineering [M.C.M., D.C., T.J.W.], Brown University, Providence, Rhode Island 02919; Department of Pediatrics [K.M.T.], \\ Rhode Island Hospital, Providence, Rhode Island 02912
}

\begin{abstract}
Ventilator-associated pneumonia (VAP) is a serious and costly clinical problem affecting pediatrics today. This devicerelated infection is thought to be directly linked to the colonization of the endotracheal tube (ETT) during long-term mechanical ventilation. Because of unspecific radiographic and clinical signs, VAP is especially difficult to diagnose in the pediatric population. Treatment with antibiotics is often ineffective, and VAP is associated with high morbidity, mortality, and medical costs. The use of nanomodified coatings on ETT may provide an effective strategy to prevent biofilm formation and ETT colonization. Nanoparticles such as selenium and iron oxide have been shown to penetrate into the biofilm reaching the protected cells antibiotics often miss. Moreover, nanoetching techniques can modify the topography of the ETT surface interfering with bacterial adhesion. This review seeks to examine the antimicrobial properties of both nanoparticles and nanomodified surfaces and to characterize their effectiveness at reducing bacterial colonization on ETT. (Pediatr Res 67: 500-504, 2010)
\end{abstract}

$\mathrm{N}$ anotechnology is the control of matter at the atomic, molecular, and supramolecular scale. Nanomaterials, or materials (such as particles, fibers, tubes, grains, etc.) with at least one dimension in the range of $1-100 \mathrm{~nm}$, can be metals, ceramics, polymers, or composites thereof. These materials exhibit unique properties because of their size and significantly greater surface areas, which can influence numerous properties including material conductivity, magnetic properties, surface energy, mechanical properties, catalytic properties, etc.

An ongoing area of this research includes the interaction between synthetic nanoscale materials and living tissues. Micro- and nanoscale building blocks form the foundation for cells and tissues within the human body. It is thought that the difference in the activity of some cells on nanomodified surfaces is because of the ability of these materials to mimic the natural dimensions of constituents of biological tissues. Another important factor in this interaction is the unique surface energetics of nanomaterials because of their significantly greater surface areas compared with conventional, micron-structured materials. Such changes in surface energy undoubtedly influence initial protein interactions that are important for mediating bacteria and nonbacteria cell adhesion. Specifically, one of the first steps within the process of cell adhesion is the association of proteins adsorbed on implant

Received November 12, 2009; accepted January 4, 2010.

Correspondence: Thomas J. Webster, Ph.D., Division of Engineering and Department of Orthopaedics, Brown University, Providence, RI 02917; e-mail: thomas_webster@brown.edu

Supported, in part, by the Rhode Island STAC Fund. surfaces to cell membrane receptors. The large surface to volume ratio characteristic of nanomaterials has been shown to affect this association to inhibit bacteria attachment and promote nonbacterial cell (such as osteoblasts, smooth muscle cells, endothelial cells, chondrocytes, etc.) adhesion (1). The type, concentration, conformation, and bioactivity of proteins adsorbed onto a material depend on its topographical (roughness), chemical, physical (charge and hydrophilicity), and mechanical (stiffness) properties, all of which can be easily influenced by nanotechnology (2). Surface properties, including topography, also effect bacterial adhesion and may play an important role in the initial stages of biofilm formation $(3,4)$.

Because of the above reasons, a vast amount of applications exist for nanomodified implant surfaces in pediatrics including antiinfection, tissue regeneration, drug delivery, and biosensing. Since the tissue growing properties of nanomaterials has been known for quite some time, this review will concentrate on the antimicrobial properties of both nanoparticles and nanomodified surfaces because of their number of applications within pediatric medicine. It will outline the prevalence of device-related infections within the pediatric population, describe improvements that can be attained by nanomodification, and will finish by examining methodologies for evaluating such materials in vitro.

\section{Nosocomial Infections in Pediatrics}

The prevention of nosocomial infections, especially ventilator-associated pneumonia (VAP), is of great importance to pediatric intensive care units (ICUs). Nosocomial infections, more commonly known as hospital acquired infections (HAIs), are estimated to occur in 5\% of all acute-care hospitalizations and result in increased mortality rates within this risk population. In addition, these deleterious conditions can be costly for both patients and hospitals, amounting to $\sim 6.7$ billion dollars per year in the United States (5). Device-related infections such as urinary tract infections and VAP affect $30 \%$ and $28 \%$, respectively, of patients who use these devices. Within pediatric ICUs, the most common HAIs are bloodstream infections followed closely by VAP (28 to $21 \%$ of all HAI, respectively) (6). The two pathogens most commonly associated with VAP are Pseudomonas aeruginosa (P. aeruginosa) and Staphylococcus aureus (S. aureus). ${ }^{1}$

Abbreviations: ETT, endotracheal tube; HAI, hospital acquired infection; SCET, silver-coated endotracheal tubes; VAP, ventilator-associated pneumonia 
VAP presents a particular intractable problem to the pediatric ICU, because it is often difficult to distinguish pneumonia from other respiratory conditions common in mechanically ventilated patients (7). This is especially true in ventilated neonates. Radiographic and clinical criteria for the diagnoses of VAP are often unspecific, causing delays in the targeted treatment and increasing the use of a broad spectrum of antibiotics. Specifically, tracheal colonization must be differentiated from lower respiratory infection. Cultures taken from patients with suspected pneumonia often yield false positives detecting benign bacteria colonization or false negatives missing the active area of bacteria infection. More than half of patients diagnosed with clinical VAP have negative cultures (8). The diagnosis of VAP in pediatric patients is hindered further by the lack of VAP studies in children and infants.

One of the main sources of bacterial colonization within the airway is the endotracheal tube (ETT) (9). As with any other medical device implanted within the body, the ETT is exposed to harmful pathogens. ETTs present a special concern to clinicians, because these tubes provide a conduit from the outside environment to the more sterile area of the lungs by impairing some of the body's natural defenses such as coughing and muscociliary motion of the trachea. Moreover, injury to the epithelial cells of the trachea can result from the movement of the tube in the airway or even from the suction of secretions during nursing care causing "opportunistic adherence" of bacteria to the airway. Chronic airway inflammation can also result from ETT insertion. Inflammation releases proteases that digest fibronectin. Fibronectin bound to the buccal epithelial surface has been shown to inhibit binding of certain Gram-negative bacteria. Fibronectin is an oposonin, mediating phagocytosis by macrophages. Recently, the gel phase of mucus in the trachea has been shown to have high levels of fibronectin and this glycoprotein is thought to aid in the mechanical removal of bacteria (2). ETT can also increase airway secretions. These increased secretions create a substrate for $P$. aeruginosa, a bacteria that binds to receptor sites on the mucus (10).

One of the key challenges to inhibit bacterial growth on ETTs occurs when certain bacteria exude an exopolysaccharide that adheres bacteria together. Bacteria in this type of extracellular matrix (known as a biofilm) are especially resistant to both antibiotics and the immune system of the patient. In such biofilms, antibiotics cannot penetrate through the extracellular matrix and cell wall effectively shielding the interior of the colony. Bacteria also must protect themselves from the innate immune system of the host organism. This is achieved through either electrostatic repulsion or proteolysis of the antimicrobial peptides of host cells, thus, preventing phagocytotic action within the biofilm. Ventilation through infected ETTs, or even condensation of humidified air can break off portions of the biofilm bringing bacteria deeper into the lungs and spurring growth on other areas of the tube (11).

Nanoparticles and nanomodified surfaces could present a unique solution to the persistent problems of VAP. The greatest numbers of bacteria in a biofilm are present at a depth of about $10 \mu \mathrm{m}$. Particle penetration into the biofilm is dependent on diffusion and is inversely related to their size while plasma clearance decreases local nanoparticle concentration. Nanoparticles are perfectly suited for biofilm penetration because they are small enough to penetrate the biofilm but large enough to have a long plasma half life. In addition, reducing the effectiveness of bacterial adhesion on the surface of an ETT could reduce both biofilm formation and bacterial contamination within the tube.

\section{Antimicrobial Properties of Nanomodified ETT}

\section{Nanoparticles}

Nanomodified surfaces have been shown to reduce bacterial growth and biofilm formation on ETTs. For example, silvercoated endotracheal tubes (SCET), which have been heavily studied, have been shown to cause a statistically significant reduction in the incidence of VAP among mechanically ventilated patients (12-14). Silver particle sizes on these tubes range from 3 to $18 \mathrm{~nm}$ with the average particle size $\sim 12 \mathrm{~nm}$. The most promising results of these ETT are their reduction of VAP in patients with highly resistant bacteria such as multiple resistant $S$. aureus. However, SCET remain cost prohibitive and cannot be used for most patients, mainly because of their expensive silver coating. Thus, these tubes are currently recommended for high-risk patients with a clinical diagnosis of VAP and are not generally used.

A number of other nanotechnologies, such as nanoparticles, have been evaluated for their antimicrobial properties. These particles can be coated on the surface of medical devices (such

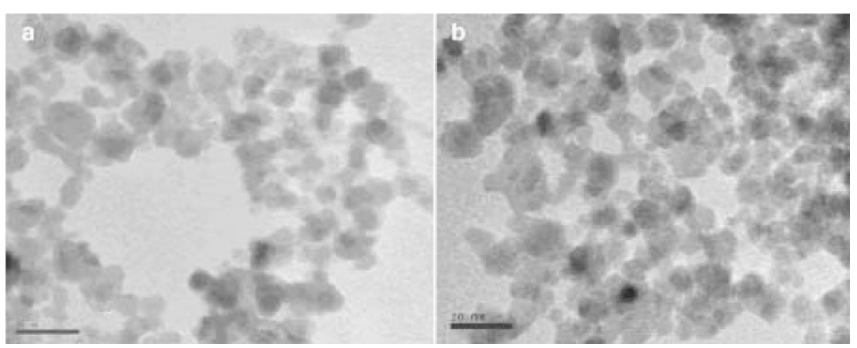

Figure 1. Transmission electron microscope (TEM) images: (a) $\gamma-\mathrm{Fe}_{2} \mathrm{O}_{3}$ and $(b) \mathrm{Fe}_{3} \mathrm{O}_{4}$. Scale bars $=20 \mathrm{~nm}$. Reprinted from Tran $\mathrm{N}$ et al., Effects of iron oxide magnetic nanoparticles on osteoblast proliferation, 2009 35th Annual Northeast Bioengineering Conference, 3-5 April 2009, Cambridge, MA; Copyright (C) 2009 IEEE, with permission.

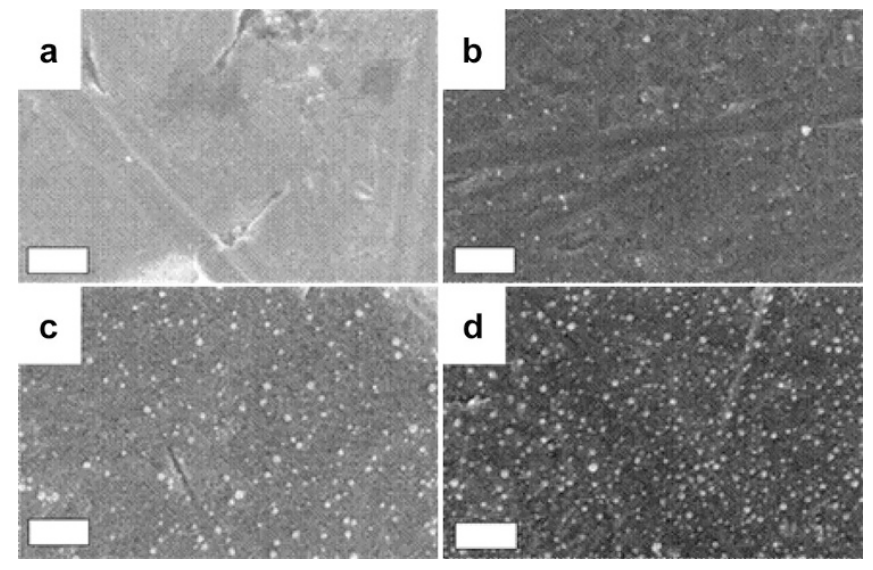

Figure 2. SEM images of uncoated $\mathrm{Ti}(a)$ and Ti coated with low (b), medium $(c)$, and high $(d)$ doses of selenium. Bars $=500 \mathrm{~nm}$. 


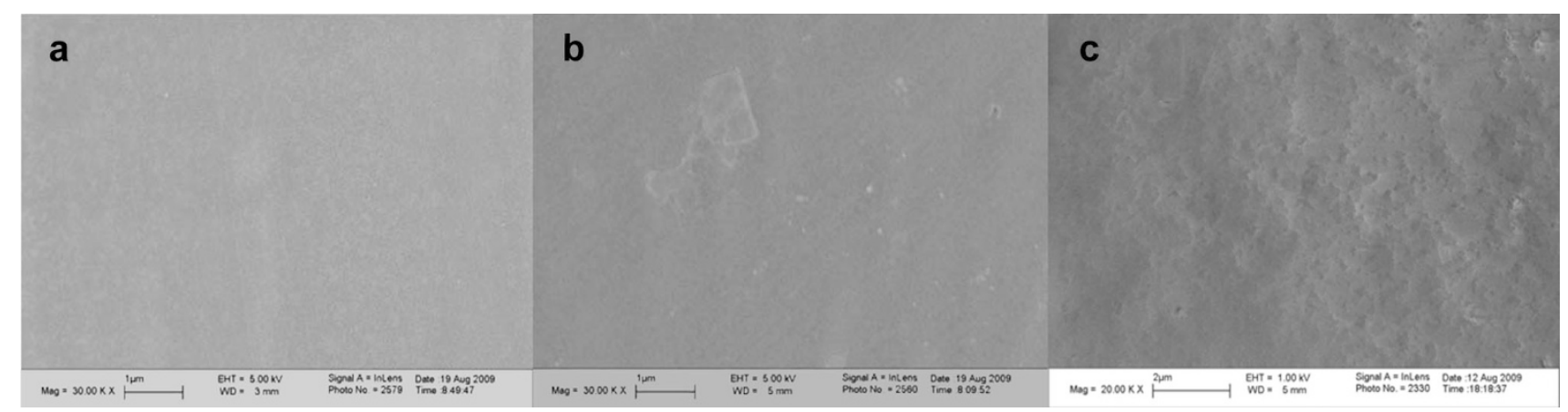

Figure 3. SEM images of nanomodified PVC ETTs: (a) Control: Magnitude $\times 30 \mathrm{~K}(b)$ Nano-C: Magnitude $\times 30 \mathrm{~K}(c)$ Nano-R: Magnitude $\times 20 \mathrm{~K}$.

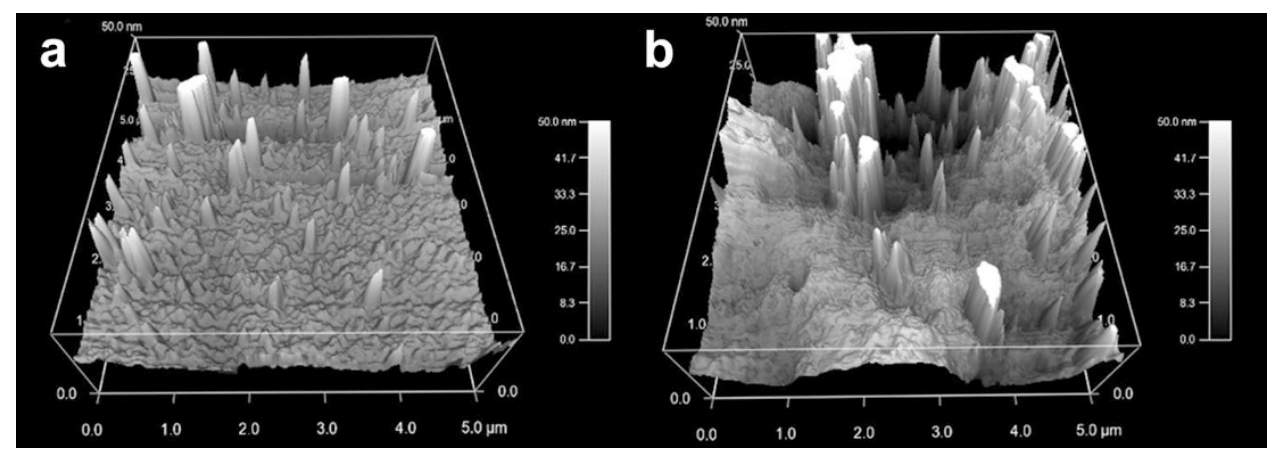

Figure 4. AFM images of nanomodified PVC ETTs. (a) Nano-R and (b) Nano-C.

as ETTs) reducing both colonization and biofilm formation. The first such particles discussed here are superparamagnetic iron oxide nanoparticles (Fig. 1), which were first evaluated in S. epidermidis cultures. Optical density (OD) studies showed decreased $S$. epidermidis numbers at all time points from 12 to $48 \mathrm{~h}$ when cultured with $100 \mu \mathrm{g} / \mathrm{mL}, 1 \mathrm{mg} / \mathrm{mL}$, and $2 \mathrm{mg} / \mathrm{mL}$ of superparamagnetic iron oxide nanoparticles (15). It is thought that $P$. Aeruginosa would also be affected by iron nanoparticles, because biofilms grown from strains of $P$. Aeruginosa found in the sputum of cystic fibrosis patients have been disrupted and cleared in flow chamber tests by switching to iron-rich medium (15).

The antimicrobial properties of selenium nanoparticles have also been evaluated for ETT applications. Selenium has been studied extensively because of its anticarcinogenic properties. However, in addition to its effect on cancer cells, nano selenium has been shown to inhibit the growth of S. epidermidis while having a positive effect on the surrounding osteoblast cells (16). These characteristics make selenium a beneficial coating for many medical devices including ETT (Fig. 2). Selenium is thought to kill bacteria by forming superoxide radicals. Selenium molecules collide with oxygen molecules within the body and then react with these molecules forming superoxide radicals. These superoxide radicals can then collide with bacteria and kill them. Healthy cells in the body remain unaffected by these free radicals because their extraordinarily short half life, $<65$ nanoseconds, only allows them to travel about the distance of half a bacterium from any device (17). Thus, selenium has been used as a coating for many medical devices because it provides a unique solution to bacterial colonization using a mineral commonly found in the diet (18). Currently, antimicrobial selenium coatings are being

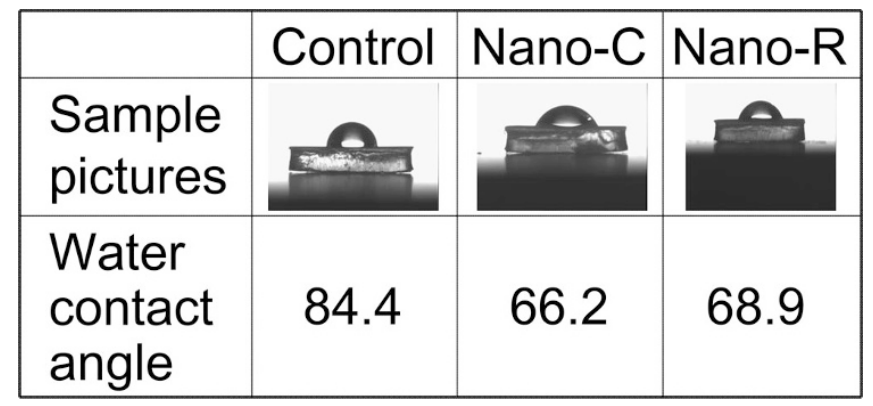

Figure 5. Contact angles of nanomodified ETTs.

evaluated on catheters, contact lenses, and artificial lenses implanted within the eye. Preliminary results suggest that nano selenium might also be effective in the inhibition of $S$. Aureus biofilms on ETT.

\section{Nanomodified surface: bacterial lipase ETT etching}

Another method of using nanotechnology to improve pediatric medicine has concentrated on increasing PVC ETT roughness at the nanoscale (Figs. 3 and 4). Nanoroughened PVC tubes have been created by soaking the PVC tubes in a $0.11 \%$ solution of bacterial lipase, which degrades the ester linkages of the surface resulting in nanoscale etching. The bacterial lipases used in this process were $C$. cilindracea (Nano-C) and R. arrhisus (Nano-R). SEM images of the nano features found on these ETT can be seen in Figure 3. Figure 4 shows atomic force microscope images of these nanomodified PVC ETT surfaces. Other surface characteristics (such as wettability) of the ETT are also changed by the nanoetching process, specifically the hydrophilicity of the nanomodified tubes is significantly different from that of the unmodified ETT, as 

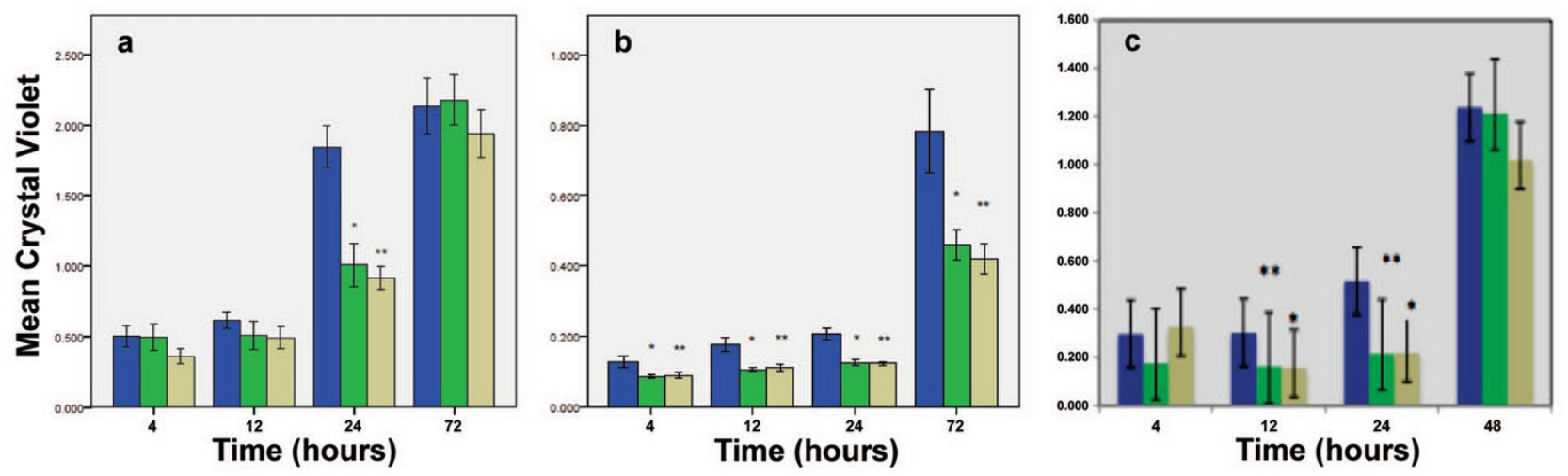

Figure 6. Comparison of bacterial concentrations on, untreated (blue), nano-C (green), and nano-R (tan), PVC ETT. (a) Decreased P. aeruginosa colonization on nanomodified PVC in TSB media. Error bars $\pm 1 \mathrm{SE}, * p<0.05 * * p<0.05$ compared with controls, $n=3$. (b) Decreased $S$. Aureus colonization on nanomodified PVC in TSB media. Error bars $\pm 1 \mathrm{SE}, * p<0.05 * * p<0.05$ compared with controls, $n=3$. (c) Decreased P. aeruginosa colonization on nanomodified PVC in saliva media. Error bars $\pm 1 \mathrm{SE},{ }^{*} p<0.05 * * p<0.05$ compared with controls, $n=3$.

seen in Figure 5 (Cheng D et al., Formation of nano surfaces on endotracheal tubes using bacterial lipase solutions, Bioengineering Conference, 2009 IEEE 35th Annual Northeast, April 3-5, 2009, Abstract).

Most importantly nanomodification of ETT also reduces bacterial growth on these tubes when tested under static conditions. Preliminary in vitro tests inoculated tryptic soy broth (TSB) and a saliva media (19) with $P$. aeruginosa and TSB with $S$. Aureus. Samples of PVC were then immersed in the inoculated media and a control for 4, 12, 24, and $72 \mathrm{~h}$ time points. The biofilm was then quantified using a crystal violet stain. Results for the TSB and saliva media can been seen in Figure 6. Statistically significant differences between the untreated tubes and the nanomodified PVC tubes were seen at all time points for $S$. Aureus and at the 24 h-time point for $P$. aeruginosa. The mechanism for this reduction on the nanomodifed tubes is unclear but most importantly is associated with the reduced adhesion of the bacteria to the surface of the tube possibly because of altered initial protein interactions.

\section{Characterizing surfaces: In vitro models}

Although promising, the characterization of bacterial colonization of nanomodified ETT in vitro has been mainly accomplished using static media studies. However, shear flow conditions have been shown to be critical in both the structural organization of biofilms and in the adhesion properties of the bacteria themselves. Biofilms grown under higher shear stresses have been shown to be more rigid than their lower shear stress counterparts (20). VAP is thought to be a direct result of the unique hydrogel structure of the biofilm (21). Therefore, new techniques to characterize the effectiveness of ETT in vitro must be considered.

To test the effectiveness of nanomodified ETT under the conditions present within the airway, a bench top model must be constructed, which takes into account both airway flow and the continuous contamination of the oropharynx. Hartmann et al. (12) created a continuously contaminated airway system to test the effectiveness of the SCET. A pediatric airway model for ETT testing has been constructed based on this model and is shown in Figure 7. Important studies of bacteria functions under dynamic conditions on nanomodified ETT remains to be conducted, including in vivo models.

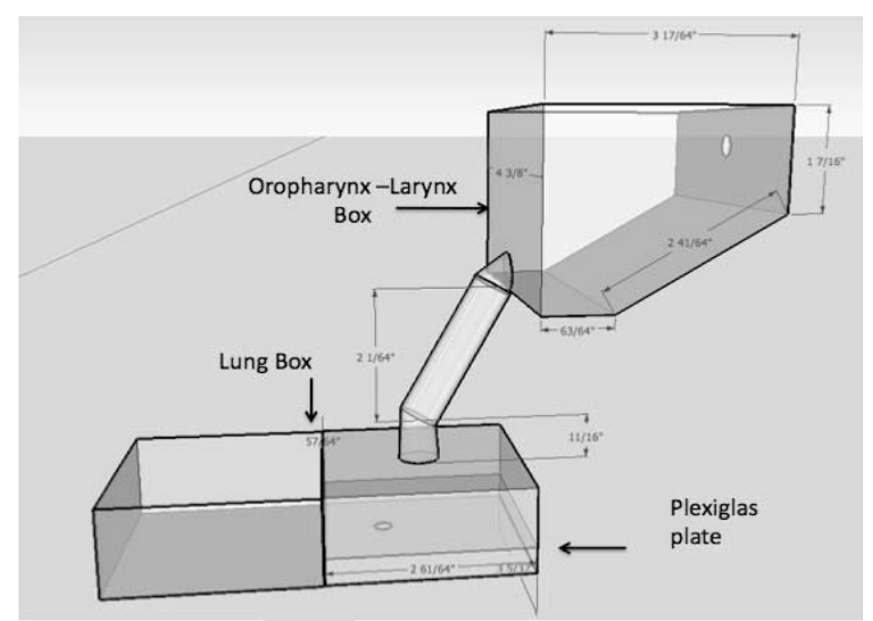

Figure 7. Plexiglas airway system schematic, which would more accurately simulate airflows ETTs are exposed to in the body.

\section{Future Directions}

The results obtained by the treatment of PVC tubes with bacterial lipases suggest that this is a viable inexpensive method to reduce bacterial colonization on ETT. Tests within the airway system will need to be performed to better characterize the mechanical properties of nanomodified ETT under more accurate airway conditions. Especially important are the airflow and humidity within the human body, which have been shown to have a critical effect on the structure and formation of biofilms. In addition, selenium and iron oxide-coated ETT should also be further analyzed as efficient but inexpensive ways to decrease infection. Further analysis needs to also examine the long-term effects of nanomodified ETTs on biofilm formation.

\section{CONCLUSIONS}

The studies mentioned in this review have shown that chemical etching with bacterial lipases can create nanorough surface features on PVC in an inexpensive but effective manner. These nanometer surface features alter the contact angles 
and, thus, the surface energy of these tubes. Nanomodified tubes have also been shown to inhibit $P$. aeruginosa growth in both TSB and saliva media. Significant reductions were seen at the 12 and 24 h-time points suggesting that these nanomodified tubes interfere with bacterial adhesion. In addition, studies with $S$. Aureus showed significantly reduced growth on ETT at all time points. OD studies with $S$. epidermidis showed decreased levels of the bacteria at all time points from 12 to $48 \mathrm{~h}$ when cultured with superparamagnetic iron oxide nanoparticles. These results suggest that nanomodified surfaces have antimicrobial properties resulting from nanotopographic interference with adhesion or free radical destruction of bacteria close to the ETT and could be useful to prevent HAIs. Further studies should focus on the influence of dynamic conditions on nanomodified ETT to gain a better understanding of their effectiveness in vivo.

\section{REFERENCES}

1. Klein J 2007 Probing the interactions of proteins and nanoparticles. Proc Natl Acad Sci USA 104:2029-2030

2. Liu H, Webster TJ 2006 Nanomedicine for implants: a review of studies and necessary experimental tools. Biomaterials 28:354-369

3. Lichter JA, Thompson MT, Delgadillo M, Nishikawa T, Rubner MF, Van Vliet KJ 2008 Substrata mechanical stiffness can regulate adhesion of viable bacteria. Biomacromolecules 9:1571-1578

4. Diaz C, Cortizo MC, Schilardi PL, Saravia SG, Mele MA 2007 Influence of the nano-micro structure of the surface on bacterial adhesion. Mater Res 10:11-14

5. Martone WJ, Jarvis WR, Culver DH, Haley RW 1993 Incidence and nature of endemic and epidemic nosocomial infections. In: Bennett JV, Brachman PS (eds) Hospital Infections. Little Brown, Boston, MA, pp 557-596
6. Richards MJ, Edwards JR, Culver DH, Gaynes RP 1999 Nosocomial infections in pediatric intensive care units in the United States. National Nosocomial Infections Surveillance System. Pediatrics 103:e39

7. Baltimore RS 2003 The difficulty of diagnosing ventilator-associated pneumonia. 112:1420-1421

8. Bahrani-Mougeot FK, Paster BJ, Coleman S 2007 Molecular analysis of oral and respiratory bacterial species associated with ventilator-associated pneumonia. J Clin Microbiol 45:1588-1593

9. Koerner RJ 1997 Contribution of endotracheal tubes to the pathogenesis of ventilator-associated pneumonia. J Hosp Infect 35:83-89

10. Carsons SE 1989 Fibronectin in Health and Disease. CRC Press Inc., Boca Raton, FL

11. Marini JJ, Slutsky A 1998 Physiological Basis of Ventilatory Support. Marcel Dekker, New York, NY

12. Hartmann M, Guttmann J, Muller B, Hallmann T, Geiger K 1999 Reduction of the bacterial load by the silver-coated endotracheal tube (SCET) a laboratory investigation. Technol Health Care 7:359-370

13. Kollef MH, Afessa B, Anzueto A, Veremakis C, Kerr KM, Margolis BD, Craven DE, Roberts PR, Arroliga AC, Hubmayr RD, Restrepo MI, Auger WR, Schinner R; NASCENT Investigation Group 2008 Silver-coated endotracheal tubes and incidence of ventilator-associated pneumonia: the NASCENT randomized trial. JAMA 300:805-813

14. Roe D, Karandikar B, Bonn-Savage N, Gibbins B, Roullet JB 2008 Antimicrobial surface functionalization of plastic catheters by silver nanoparticles. J Antimicrob Chemother 61:869-876

15. Taylor EN, Webster TJ 2009 The use of superparamagnetic nanoparticles for prosthetic biofilm prevention. Int J Nanomedicine 4:145-152

16. Webster TJ, Siegel RW, Bizios R 2000 Enhanced functions of osteoblasts on nanophase ceramics. Biomaterials 21:1803-1809

17. de Leiris J 2003 Biochemistry of free radicals. Heart Metab 19:40-44

18. Clark LC, Dalkin B, Krongrad A, Combs GF Jr, Turnbull BW, Slate EH, Witherington R, Herlong JH, Janosko E, Carpenter D, Borosso C, Falk S, Rounder J 1998 Decreased incidence of prostate cancer with selenium supplementation: results of a double-blind cancer prevention trial. Br J Urol 81:730-734

19. Söderholm KJ, Mukherjee R, Longmate J 1996 Filler leachability of composites stored in distilled water or artificial saliva. J Dent Res 75:1692-1699

20. Stoodley P, Jacobsen A, Dunsmore BC, Purevdorj B, Wilson S, Lappin-Scott HM, Costerton JW 2001 The influence of fluid shear and AICI3 on the material properties of Pseudomonas aeruginosa PAO1 and Desulfovibrio sp. EX265 biofilms. Water Sci Technol 43:113-120

21. Ghannoum MA, O’Toole GA 2004 Microbial Biofilms. ASM Press, Washington, DC 